\title{
Hydrodynamic and Dynamic Analysis to Determine the Longitudinal Hydrodynamic Coefficients of an Autonomous Underwater Vehicle
}

\author{
Le Quang*, Phan Anh Tuan, Pham Thi Thanh Huong \\ Hanoi University of Science and Technology, No. 1, Dai Co Viet, Hai Ba Trung, Hanoi, Viet Nam \\ Received: June 07, 2020; Accepted: November 12, 2020
}

\begin{abstract}
A useful tool for understanding the performance of an Autonomous Underwater Vehicle (AUV) is a dynamic simulation of the motions of the vehicle. To perform the simulation, the hydrodynamic coefficients of the vehicle must be first provided. These coefficients are specific to the vehicle and provide the description of hydrodynamic forces and moments acting on the vehicle in an underwater environment. This paper provides a method for the calculation and evaluation of the hydrodynamic coefficients of an AUV. The presence methodology is therefore one useful tool for determining an underwater vehicle's dynamic stability. The calculated values have been compared with experimental results of a torpedo shape. It was concluded that the methods could calculate accurate values of the hydrodynamic coefficients for a specific AUV shape with its elliptical nose
\end{abstract}

Keywords: Stability, Autonomous Underwater Vehicles (AUV), simulation, hydrodynamic coefficients

\section{Introduction}

During the underwater vehicle scheme design period, the simulation and evaluation of the stability of submarine is an important task. Simulation of the motion of an underwater vehicle requires the numerical solution of six-coupled non-linear differential equations. Three of these equations describe the translational motions of the vehicle, the remaining three equations describe rotational motions of the vehicle about some fixed point on the body [1]. This fixed point is usually taken to be either the centre of mass $(\mathrm{CM})$ or the centre of buoyancy $(\mathrm{CB})$ of the vehicle. Detailed derivations and discussions of these equations of motion can be found in many references [2], [3]. Traditionally, the methods to predict the hydrodynamic derivatives of underwater vehicles could be classified into three types: the semi-empirical method, the potential flow method, and the captive-model experiments including the oblique towing tests, the rotating arm experiments and the Planar Motion Mechanism (PMM) [1].

With the semi-empirical method, the complicated underwater vehicle shape usually could not be taken into full account. The potential theory could predict the inertial hydrodynamic coefficients satisfactorily, but with the viscous terms neglected [3]. The PMM experiment may be the most effective way, but it requires special facilities and equipment and it is both time-consuming and costly [3], as not economical at the preliminary design stage.

*Corresponding author: Tel.: (+84) 913.223.160

Email: quang.le@hust.edu.vn
This paper shows the results by using the semiexperimental method (used U.S Air Force DATCOM method). This method is based on the techniques developed in the aeronautical industry. The calculated values were then compared with CFD results and other data available [2].

\section{Equation of AUV Motion}

The equations of motion for a submarine are similar to those for an aircraft, they include all six degrees of freedom [1],[5],[7]. For a submarine, it is normal to take the origin as the longitudinal centre of gravity (LCG), rather than midships, as this simplifies the equations, and for a submarine, this position is fixed (unlike for a surface ship). The axis system used is shown in the notation in Table 1.

Table 1. Notation

\begin{tabular}{|l|c|c|c|}
\hline & Position & Velocity & Force/Moment \\
\hline Surge & $x$ & $u$ & $X$ \\
\hline Sway & $y$ & $v$ & $Y$ \\
\hline Heave & $z$ & $w$ & $Z$ \\
\hline Roll & $\phi$ & $p$ & $K$ \\
\hline Pitch & $\theta$ & $q$ & $M$ \\
\hline Yaw & $\psi$ & $r$ & $N$ \\
\hline Appendage & $\delta$ & & \\
\hline Propulsion & & $n$ & \\
\hline
\end{tabular}


The equations of motion are based on Newton's Second Law: Force $=$ Mass $\times$ Acceleration. In this case, the force, the left-hand side of the equation, is the hydrodynamic force acting on the submarine, and the right-hand side is the rigid body dynamics, the right-hand side of the equation is given as follows:

$$
\begin{aligned}
& X=m\left[\begin{array}{l}
\bullet \\
u-v r+w q-x_{G}\left(q^{2}+r^{2}\right)+y_{G}(p q-r)+z_{G}(p r+q)
\end{array}\right] \\
& Y=m\left[\begin{array}{l}
\bullet-w p+u r+x_{G}(q p+r) \\
\dot{0}
\end{array} y_{G}\left(r^{2}+p^{2}\right)+z_{G}(q r-\dot{p})\right] \\
& \left.Z=m\left[\begin{array}{l}
\bullet-u q+v p+x_{G}(r p-q \\
w
\end{array}\right)+y_{G}(r q+\dot{p})-z_{G}\left(p^{2}+q^{2}\right)\right] \\
& K=I_{x x} \dot{p}^{\bullet}\left(I_{x x}-I_{y y}\right) q r-(\stackrel{\bullet}{r+p q}) I_{z x}+\left(r^{2}-q^{2}\right) I_{y z}+(p r-q) I_{x y} \\
& +m\left[y _ { G } \left(\begin{array}{l}
\bullet-u q+v p)-z_{G}(\dot{v}-w p+u r) \\
\bullet
\end{array}\right.\right. \\
& M=I_{y y} \dot{\bullet}_{+}\left(I_{x x}-I_{z z}\right) r p-(\dot{p}+q r) I_{x y}+\left(p^{2}-r^{2}\right) I_{z x}+(q p-r) I_{y x} \\
& -m\left[x_{G}(w-u q+v p)-z_{G}\left(\mathfrak{u}^{\bullet} v r+w q\right)\right] \\
& N=I_{z z} r+\left(I_{y y}-I_{x x}\right) p q-(\dot{q}+r p) I_{y x}+\left(q^{2}-p^{2}\right) I_{x y}+(r q-p) I_{z x} \\
& +m\left[x_{G}(\dot{v}-w p+u r)-y_{G}(\dot{\bullet}-u q+v p)\right]
\end{aligned}
$$

$X, Y, Z, K, M$, and $N$ are the total hydrodynamic surge, sway, and heave forces, and roll, pitch, and yaw moments respectively. If these hydrodynamic forces and moments can be determined as functions of time for a maneuvering submarine, then the movement of AUV can be simulated. In addition, if the effects of geometry on these forces and moments are understood then this can be used to assist in the design of the submarine. The expressions for the forces and moments then take the form:

$$
\begin{aligned}
& X=X_{0}+X_{u} u+X_{v} v+X_{w} w+X_{p} p+X_{p} q+X_{r} r \\
& Y=Y_{0}+Y_{u} u+Y_{v} v+Y_{w} w+Y_{p} p+Y_{p} q+Y_{r} r \\
& Z=Z_{0}+Z_{u} u+Z_{v} v+Z_{w} w+Z_{p} p+Z_{p} q+Z_{r} r \\
& L=L_{0}+L_{u} u+L_{v} v+L_{w} w+L_{p} p+L_{p} q+L_{r} r \\
& M=M_{0}+M_{u} u+M_{v} v+M_{w} w+M_{p} p+M_{p} q+M_{r} r \\
& N=N_{0}+N_{u} u+N_{v} v+N_{w} w+N_{p} p+N_{p} q+N_{r} r^{2}
\end{aligned}
$$

In equations the subscript notation represents partial differentiation, so that $X_{u}=\frac{\partial X_{u}}{\partial t}$ and the zero subscript refers to conditions in the assumed reference state. The partial derivatives are known as hydrodynamic coefficients, hydrodynamic derivatives, or stability derivatives, and are evaluated at the reference condition.

There are 36 hydrodynamic coefficients which could be evaluated to describe the dynamics of the vehicle. If the vehicle has certain symmetries however then many of these coefficients are zero. For example, if the $x-z$ plane is a plane of symmetry so that the vehicle has Left/Right symmetry, then terms such as $Y u, Y w, L u, L w$, etc. will all be zero. $Y u$, for example, is the contribution to the component of force in the $y$-direction due to motion in the $\mathrm{x}$ direction. For a body with Left/Right symmetry, it is easy to see that this contribution will always be zero.

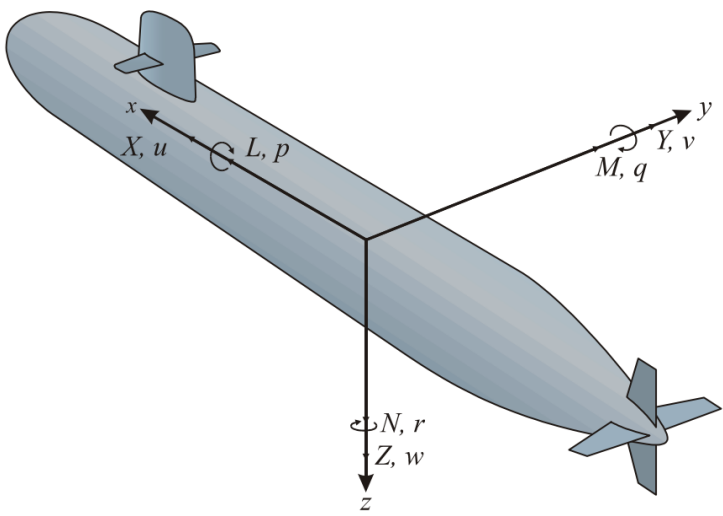

Fig. 1 Coordinate system used fo AUV

Some authors [2],[4] had concluded that the non-zero coefficients for axe-symmetric AUVs are $X u, \quad X v, X w, Z w, Z q, \quad M w$ and $M q$ in the longitudinal plane, and $Y v, Y r, N v$, and $N r$ in the lateral plane.

In [6] discussing aerodynamic derivatives for aeroplanes, notes that for symmetric aircraft the derivatives of the asymmetric or lateral forces and moments, $Y, L$, and $N$, with respect to the symmetric or longitudinal motion variables $u, w$, and $q$, will be zero. This implies that $Y u, Y w, Y q, L u, L w, L q, N u$, $N w$, and $N q$ are zero for aircraft, and bodies which exhibit similar symmetry properties. All the derivatives of the symmetric forces $(X, Z)$ and moments $(M)$ with respect to the asymmetric variables ( $v, p$ and $r$ ) can be neglected. This implies that $X v, X p, X r, Z v, Z p, Z r, M v, M p$ and $M r$ are zero. This result also could be applied to an AUV

For the longitudinal coefficients, $X u, Z w, Z q$, $M w$ and $M q$ are important while $Z u$ and $M u$ are less important. The other longitudinal coefficients such as $Y v, Y r, N v$, and $N r$ are neglected because of the symmetry in geometry of the AUV. 


\section{Determination of hydrodynamic coefficients}

In the present work, the hydrodynamic stability derivatives are determined through DATCOM method [3], combined with CFD simulation. Peterson uses the DATCOM method for the calculation of the hydrodynamic coefficients $Z_{w, B}^{\prime} ; M_{w, B}^{\prime} ; Z_{q, B}^{\prime}$ and $M_{q, B}^{\prime}$ for the bare hull, and $Z_{w, B}^{\prime}$ and $M_{w, B}^{\prime}$ for the bare hull plus horizontal tail configuration. This is presented below. The prime notation above indicates a dimensionless coefficient, and Peterson accepted the convention that all derivatives are nondimensional with respect to the body cross-sectional area $S b$ and the body length $l$

Calculation of $Z_{w, B}^{\prime}$ : Peterson's expression [2] for $\mathrm{Zw}$ for the body alone is:

$$
Z_{w, B}^{\prime}=-\left(\frac{S_{b}}{l^{2}}\right)\left[C_{L_{\alpha, B}}+C_{D_{0}}\right]
$$

where $C_{L_{\alpha, B}}$ is the body alone lift-curve slope and $C_{D_{0}}$ is the drag coefficient at zero lift. Given by CFD simulation.

Calculation of $M_{w, B}^{\prime}$ : The pitching moment/ angle of attack is calculated in DATCOM by applying a viscous correction to the Munk moment [2]

$$
C_{m_{\alpha, \beta}}=\frac{2\left(k_{2}-k_{1}\right)}{S_{b} l} \int_{0}^{l} \frac{d S}{d x}\left(x_{m}-x\right) d x
$$

where $x_{m}$ is defined as being the distance from the nose to the moment reference center and $l_{v}$ is the axial location of separation. The final expression for the pitching moment is then:

$$
M_{w, B}^{\prime}=\left(\frac{S_{b}}{l^{2}}\right) C_{\alpha, \beta}
$$

Calculation of $Z_{q, B}^{\prime}$ : Peterson considers only the contribution of the bare hull to $\mathrm{Z}_{\mathrm{q}}$ and $\mathrm{M}_{\mathrm{q}}$ [2].

$$
C_{L_{q, B}}=C_{L_{\alpha, B}}\left(1-\frac{x_{m}}{l}\right)
$$

where $x_{m}$ is the distance from the nose to the moment reference center. $Z_{\mathrm{q}}$ is then given by

$$
Z_{q, B}^{\prime}=-\left(\frac{S_{b}}{l^{2}}\right) C_{L_{q, \beta}}
$$

3.4 Calculation of $M_{q, B}^{\prime}$ : The pitching moment/pitch rate curve slope [2]:

$$
\begin{aligned}
& C_{m_{q, B}}=C_{m} \frac{\left(1-\frac{x_{m}}{l}\right)^{2}-\frac{V}{S_{t b} l}\left(\frac{l_{c}}{l}-\frac{x_{m}}{l}\right)}{\left(1-\frac{x_{m}}{l}\right)-\frac{V}{S_{t b}}} \\
& M_{q, B}^{\prime}=-\left(\frac{S_{b}}{l^{2}}\right) C_{m_{q, \beta}}
\end{aligned}
$$

where $l_{\mathrm{c}}$ is the distance from the nose to the entre of buoyancy and $S_{t b}$ is the cross sectional area of the truncated base. The tail-alone lift-curve slope is calculated using the following [2]:

$$
C_{L_{\alpha, T}}=\frac{2 \pi A R}{2+\sqrt{4+(A R)^{2}\left(1+\tan ^{2} \lambda_{c / 2}\right)}} \frac{S_{t}}{S_{b}}
$$

where $\mathrm{AR}$ is the aspect ratio of the tail, $\lambda \mathrm{c} / 2$ is the sweep angle at the half-chord line, and $S t$ is the total tail planform area. The expression for the combined body/tail lift-curve slope is then

$$
C_{L_{\alpha, \text { wing }}}=\frac{2 \pi A R}{2+\sqrt{4+4 \pi^{2}(A R)^{2}\left(1+\tan ^{2} \lambda_{c / 2}\right) / C_{L \alpha}^{2}}}
$$

For $C_{L_{\alpha, T}}$ is actually an approximate expression which has been derived using thin wing theory, $C_{L \alpha}=2 \pi \alpha$. The interference of the fuselage with the wing [8], as well as the contribution of the fuselage itself to the lift, is taken into account using the following expression:

$$
C_{L_{\alpha, w f}}=K_{w f} C_{L, w i n g}
$$

where $K_{w f}$ is a correction factor which has the form

$$
K_{w f}=1+0.025 \frac{d f}{b}-0.25\left(\frac{d f}{b}\right)^{2}
$$

where $d_{f}$ is the maximum fuselage diameter and $b$ is the wing span [6], [7]. 


$$
\begin{gathered}
C_{L \alpha}=\frac{\left.C_{L \alpha}\right|_{A R=\infty}}{1+\frac{\left.C_{L \alpha}\right|_{A R=\infty}}{\pi A R}} \\
C_{D}=C_{D_{0}}+\frac{C_{L}^{2}}{\pi A R}
\end{gathered}
$$

where $A R$ is the aspect ratio, $C_{L}$ is the wing lift coefficient

\section{The AUV Model}

To identify hydrodynamic coefficients of the AUV, a model of AUV is used in this study. The principal parameters of AUV are shown in Table 2.

Table 2. Principal technical parameters of AUV

\begin{tabular}{|l|l|r|}
\hline \multicolumn{1}{|c|}{ Content } & \multicolumn{1}{c|}{$\begin{array}{c}\text { Symbol } \\
\text { (Unit) }\end{array}$} & \multicolumn{1}{c|}{ Value } \\
\hline Length & $L(\mathrm{~mm})$ & 3013 \\
\hline Height & $H(\mathrm{~mm})$ & 462 \\
\hline Diameter & $D(\mathrm{~mm}), d_{f}$ & 300 \\
\hline $\begin{array}{l}\text { Draught without water } \\
\text { in the 4 floats }\end{array}$ & $T(\mathrm{~mm})$ & 296 \\
\hline Weight & $M(\mathrm{Kg})$ & 200 \\
\hline Wingspan & $b(\mathrm{~mm})$ & 462 \\
\hline Sea water density & $\rho\left(\mathrm{kg} / \mathrm{m}^{3}\right)$ & 1035 \\
\hline Velocity & $V(\mathrm{~m} / \mathrm{s})$ & 2.0 \\
\hline Moments of inertia & $I_{x x}\left(\mathrm{kgm}^{2}\right)$ & 2.2184 \\
\hline Centre of gravity & $G(x, y, z)$ & $0,0,0$ \\
\hline Centre of buoyancy & $B(x b, y b, z b)$ & $0,0,50$ \\
\hline
\end{tabular}
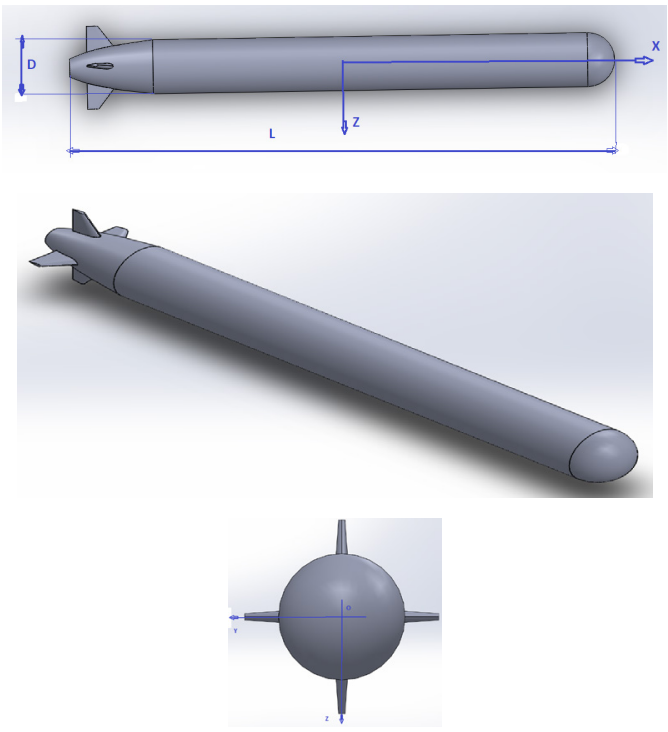

Fig. 2. Geometry of underwater vehicle

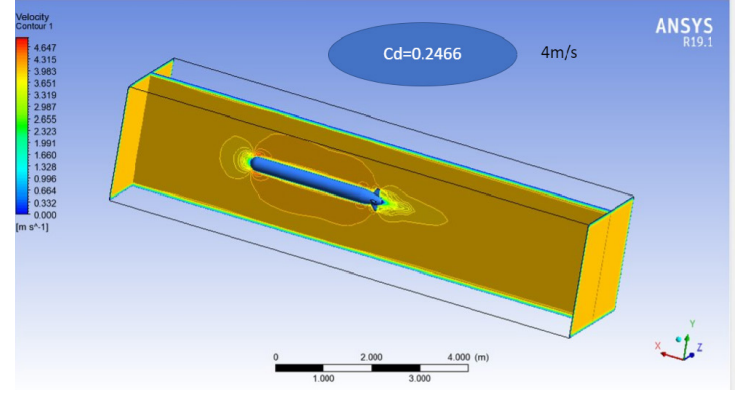

Fig. 3. Velocity contour around AUV

\section{Results and Discussions}

First, the drag and lift coefficients of AUV motion have been calculated by using CFD method. The steady state CFD was successfully applied to simulate the straight line. Reynolds-averaged NavierStokes (RANS) equations are time-averaged equations of motion for fluid flow and as an approach to solve Navier-Stokes equations [5]. Fig. 3 shows the velocity contour around AUV and for calculation of the necessary values as $C_{L}, C_{D}, C_{M}$ when using the tools above.

The AUV has been carried simulation with its velocity from $0.5 \mathrm{~m} / \mathrm{s}$ to $5.0 \mathrm{~m} / \mathrm{s}$ and the vehicle moves in infinite fluid with three different turbulent models of $k-\varepsilon$. Fig.3 shows the velocity contour around AUV for $3 \mathrm{~m} / \mathrm{s}$. Table 3 and Fig.5 show the drag and drag coefficient of the AUV whit diferent velocities. The drag coefficient of AUV is identified by using equation (16) below.

$$
C_{D}=\frac{2 F_{D}}{\rho S V^{2}}
$$

$F_{D}(N)$ - Force resistance (drag); $\rho=1035 \mathrm{~kg} / \mathrm{m}^{3}$ density of sea water, $S=0.07065 \mathrm{~m}^{2}$ - reference area (the cross perpendicular section with motion).

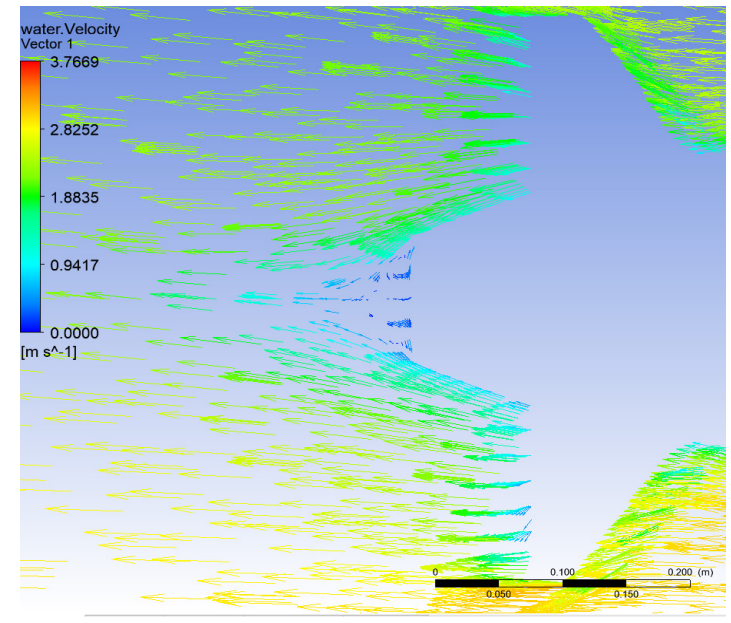

Fig. 4. Velocity contour around the aft of AUV 
Table 3. Drag/Drag coefficient in the function of velocity

\begin{tabular}{|c|c|c|}
\hline Velocity $(\mathrm{m} / \mathrm{s})$ & Drag $F_{D}(\mathrm{~N})$ & $\begin{array}{c}\text { Drag coefficient } \\
C_{D}\end{array}$ \\
\hline 0.5 & 2.22 & 0.24 \\
\hline 1.0 & 8.77 & 0.24 \\
\hline 1.5 & 18.92 & 0.23 \\
\hline 2.0 & 36.41 & 0.24 \\
\hline 2.5 & 53.92 & 0.23 \\
\hline 3.0 & 82.14 & 0.25 \\
\hline 3.5 & 115.99 & 0.26 \\
\hline 4.0 & 151.50 & 0.25 \\
\hline 4.5 & 191.75 & 0.24 \\
\hline 5.0 & 236.73 & 0.25 \\
\hline
\end{tabular}

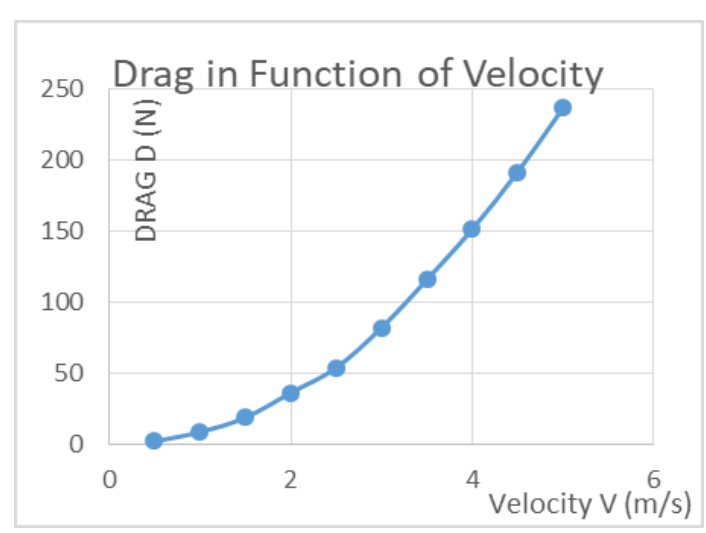

Fig. 5. Resistance of AUV

Fig. 4 shows the velocity around the aft body of AUV when the AUV moves near the seabed and for calculation of tail lift curve slope.

Using the math formulas from equations (10) to (15), the parameters of aft Hydroplanes are calculated in Table 4.

Method CFD for the calculation of the lift and drag coefficient of alone wing are clearly presented in (6) and do not need to be repeated here.

Table 4. Designed and calculated parameters of Aft Hydroplanes NACA 0012

\begin{tabular}{|l|c|c|}
\hline Parameter & Values & Remark \\
\hline Aspect ratio of the tail & 3.7 & $A R$ \\
\hline $\begin{array}{l}\text { Sweep angle at the half- } \\
\text { chord line }\end{array}$ & $20^{\circ}$ & $\lambda \mathrm{c} / 2$ \\
\hline $\begin{array}{l}\text { Total } \\
\text { tail planform area }\end{array}$ & $0.0579 \mathrm{~m}^{2}$ & $S t$ \\
\hline Tail-alone lift-curve slope & $5.63 \mathrm{rad}^{-1}$ & $C_{L \alpha}$ \\
\hline Tail-alone lift-curve slope & $3.49 \mathrm{rad}^{-1}$ & $C_{L \alpha, t}$ \\
\hline $\begin{array}{l}\text { Tail-fuselage lift-curve } \\
\text { slope }\end{array}$ & $3.79 \mathrm{rad}^{-1}$ & $C_{L \alpha, t f}$ \\
\hline Drag coefficient of tail & 0.0213 & $C_{D t}$ \\
\hline
\end{tabular}

The hydrodynamic stability derivatives are determined through DATCOM method, combined with CFD simulation. For the longitudinal coefficients note that $X u, Z w, Z q, M w$, and $M q$ were important coefficients, while $Z u$ and $M u$ were less significant. These other longitudinal coefficients are zero [8]. Table 5 shows the calculated values for the four longitudinal hydrodynamic coefficients for this AUV.

Table 5. Longitudinal hydrodynamic coefficients

\begin{tabular}{|c|c|c|}
\hline Coefficients & $\begin{array}{c}\text { Value } \\
\text { calculated }\left(\times 10^{-3}\right)\end{array}$ & $\begin{array}{l}\text { Math } \\
\text { formula }\end{array}$ \\
\hline$Z^{\prime}{ }_{W}$ & -1.79 & $(3)$ \\
\hline$M^{\prime}{ }_{W}$ & 0.882 & $(5)$ \\
\hline$Z^{\prime} q$ & -14.4 & $(7)$ \\
\hline$M^{\prime} q$ & -0.455 & $(9)$ \\
\hline
\end{tabular}

The data experimental for this AUV is not available so in order to check the correctness of this method, we use the test values obtained from documentation [2].

Table 6 shows the comparison of calculated and experimental values for Torpedo 13 done by Hyguess [2].

Table 6. Comparison of calculated and experimental values.

\begin{tabular}{|l|c|c|c|}
\hline Coefficient & $\begin{array}{c}\text { Value } \\
\text { calculated }\end{array}$ & Experiment & $\begin{array}{c}\text { Percentage } \\
\text { difference }\end{array}$ \\
\hline$Z^{\prime}{ }_{W}$ & -0.593 & -0.60 & 1.2 \\
\hline$M^{\prime}{ }_{W}$ & 0.993 & 0.99 & 0.0 \\
\hline$Z^{\prime} q$ & -0.209 & -0.20 & 5.0 \\
\hline$M^{\prime} q$ & -0.074 & -0.08 & 7.5 \\
\hline
\end{tabular}

Table 6 shows that the percentage difference between values calculated and experimental is acceptable. From there the results calculated in table 5 can be accepted.

\section{Conclusion}

A detailed description of the calculation of each of four longitudinal hydrodynamic coefficients of AUV is identified by DATCOM method, based on techniques developed in the aeronautical industry. In this paper, DATCOM method is used to calculate the hydrodynamic coefficients for an AUV with a torpedo shape.

The calculated values have been compared with experimental results of a torpedo shape. It was concluded that the methods described above could calculate accurate values of the hydrodynamic coefficients for a specific AUV shape with its 
elliptical nose, the main body is cylindrical and the aft is conical.

\section{Acknowledgments}

This work is supported by the Ministry of Science and Technology with project code: NĐT.68.RU/19.

\section{References}

[1] Martin Renilson, Submarine Hydrodynamics, Springer, ISBN 978-3-319-16184-6, 2015

[2] D.A. Jone, D.B. Clarke, I.B. Brayshaw, J.L. Barillon, B. Anderson, The Calculation of Hydrodynamic Coefficients for Underwater Vehicles, Victoria, DSTO COA, Australia, 2002

[3] Finck, R.D. "USAF Stability and Control Data Compendium" (DATCOM), Air Force Flight
Dynamics Laboratory, Wright Patterson Air Force Base, April, 1976

[4] Fossen T. I., Handbook of Marine Craft Hydrodynamics and Motion Control, Wiley, 2011.

[5] Lê Quang, Pham T.T. Hương, Tính toán các đặc tính động lực học và khảo sát ổn định chế độ hạ cánh của máy bay phản lực luyện tập loại nhỏ, Báo Tạp chí Cơ khí, Số 6(2019). 68-72

[6] Robert C. Nelson. Flight Stability and Automatic Control. 2 rd Edition, McGraw-Hill, 1984.

[7] M. Nita, D. Scholz. Estimating the Oswald Factor from Basic Aircraft Geometrical Parameters, Deutscher Luft-und Raumfahrtkongress (2012). 14-19

[8] Vepa Ranjan. Flight Dynamics, Simulation and Control for Rigid and Flexible Aircraft. CRC Press, Taylor \& Francis Group, LLC, 2015 